\title{
Aplicação da análise de regressão linear na estimativa da energia de ativação de fluxo dos ácidos oleico e palmítico
}

César Augusto CANCIAM*

*Mestre em Engenharia Química, professor lotado no Departamento Acadêmico de Engenharia Química da

Universidade Tecnológica Federal do Paraná - Câmpus Ponta Grossa, canciam@utfpr.edu.br

Recebido em: 01/02/2013 - Aprovado em: 22/05/2013 - Disponibilizado em: 15/08/2013

\section{RESUMO:}

A sensibilidade da viscosidade devido ao aumento de temperatura é indicada pela energia de ativação de fluxo, de maneira que, quanto maior for o valor da energia de ativação de fluxo, maior será a influência da temperatura sobre a viscosidade. O objetivo deste trabalho foi estimar a energia de ativação de fluxo dos ácidos oleico e palmítico entre 70 e $100^{\circ} \mathrm{C}$. Os valores da energia de ativação de fluxo foram determinados a partir da análise de regressão linear dos dados da viscosidade (estimados pela equação de Andrade e pelo modelo de GONZÁLEZ et al. (1999)) em função da temperatura. Os valores encontrados para a energia de ativação de fluxo do ácido oleico foram 22,948 kJ.mol ${ }^{-1}$ (com base na equação de Andrade) e 22,477 kJ.mol ${ }^{-1}$ (com base no modelo de GONZÁLEZ et al. (1999)). Para o ácido palmítico, os valores encontrados foram $23,152 \mathrm{~kJ} \mathrm{~mol}^{-1}$ (com base na equação de Andrade) e 23,118 kJ.mol ${ }^{-1}$ (com base no modelo de GONZÁLEZ et al. (1999)). Em todos os casos estudados, os coeficientes de correlação foram próximos da unidade, classificando a correlação linear como muito forte. Não foram encontrados valores experimentais para a energia de ativação de fluxo dos ácidos oleico e palmítico na literatura.

PALAVRAS-CHAVE: Viscosidade. Temperatura. Energia de ativação de fluxo. Estimativa. Ácidos graxos.

\section{Application of linear regression analysis to estimate the activation energy of flow of oleic and palmitic acids}

\begin{abstract}
:
The sensitivity of viscosity due to temperature rise is indicated by the activation energy of flow, so that the larger the value of the activation energy of flow, the greater the influence of the temperature on the viscosity. The objective of this work was to estimate the activation energy of flow of oleic and palmitic acids between 70 and $100^{\circ} \mathrm{C}$. The values of the activation energy of flow were determined from the linear regression analysis of data viscosity (estimated by Andrade's equation and model proposed by GONZÁLEZ et al. (1999)) as a function of the temperature. The values found for the activation energy of flow of oleic acid were $22.948 \mathrm{~kJ} \mathrm{~mol}^{-1}$ (based on Andrade's equation) and $22.477 \mathrm{~kJ}^{\mathrm{mol}}{ }^{-1}$ (based on the model proposed by GONZÁLEZ et al. (1999)). For palmitic acid, the values found were $23.152 \mathrm{~kJ}^{-\mathrm{mol}^{-1}}$ (based

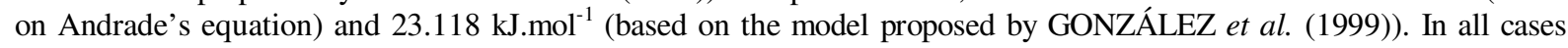
studied, the correlation coefficients were close to unity, classifying the linear correlations as very strong. There were no experimental values of the activation energy of flow of oleic and palmitic acids in the literature.
\end{abstract}

KEYWORDS: Viscosity. Temperature. Activation energy of flow. Estimate. Fatty acids.

\section{INTRODUÇÃO}

Uma das teorias que explicam a viscosidade nos líquidos é a Teoria do Estado Ativado de Eyring (1941) (RIBEIRO et al., 2005).
Essa teoria considera que um líquido é constituído por moléculas intercaladas por posições desocupadas (vacâncias). Mesmo o líquido em repouso, as moléculas movem-se a fim de ocuparem as vacâncias adjacentes a elas. A viscosidade está associada com a força que tende a se opor a esse movimento, sendo 
uma medida da fricção interna do líquido. Assim, quanto maior for à barreira potencial que uma molécula terá que vencer com o objetivo de "saltar" para a vacância adjacente, maior será a viscosidade. Essa barreira potencial é denominada de energia de ativação de fluxo (RIBEIRO et al. , 2005; SILVA et al. , 2007).

Pela Teoria do Estado Ativado de Eyring, o escoamento de um líquido pode ser considerado um processo térmico, em que as moléculas devem exceder a energia de ativação de fluxo (BRINGEL et al., 2009).

Quando se aumenta a temperatura, a energia térmica das moléculas aumenta, assim como as vacâncias. Ocorre a diminuição das forças de atração entre as moléculas, diminuindo a viscosidade. Isto é atribuído ao aumento das distâncias intermoleculares provocadas durante o aquecimento (GRANJEIRO et al., 2007; BRINGEL et al., 2009).

A dependência da viscosidade absoluta $(\mu)$ com a temperatura $(\mathrm{T})$ é modelada a partir da equação de Arrhenius (conforme a Equação 1) (FASINA e COLLEY, 2008; GIAP, 2010).

$$
\mu=\mu_{\infty} \cdot \exp \left(\frac{E_{a}}{R \cdot T}\right)
$$

Em que: $\mathrm{E}_{\mathrm{a}}$ corresponde à energia de ativação de fluxo; $\mathrm{R}$, à constante universal dos gases ideais e $\mu_{\infty}$, à viscosidade absoluta quando a temperatura tende ao infinito (GIAP, 2010).
Na Equação 1, se o valor da energia de ativação de fluxo for baixo, a viscosidade absoluta não é tão sensível à variação de temperatura (KERSTNER et al. , 2011).

A Equação 1 pode ser reescrita na forma linearizada, indicada pela Equação 2 (GRATÃO et al., 2004):

$$
\ln \mu=\left(\frac{E_{a}}{R}\right) \cdot\left(\frac{1}{T}\right)+\ln \mu_{\infty}
$$

Um gráfico de $\ln \mu$ versus $(1 / \mathrm{T})$ fornece uma reta, em que o coeficiente angular é numericamente igual à razão $\left(\mathrm{E}_{\mathrm{a}} / \mathrm{R}\right)$ e o coeficiente linear, à $\ln \mu_{\infty}$ (CANCIAM, 2008).

$\mathrm{O}$ ácido oleico $\left(\mathrm{C}_{18} \mathrm{H}_{34} \mathrm{O}_{2}\right)$ é um ácido graxo monoinsaturado constituído por dezoito átomos de carbono com uma dupla ligação começando no nono átomo de carbono contado a partir do átomo de carbono do grupo metila (BRUICE, 2006).

Já o ácido palmítico $\left(\mathrm{C}_{16} \mathrm{H}_{32} \mathrm{O}_{2}\right)$ é um ácido graxo saturado de cadeia longa (BRUICE, 2006).

Os ácidos graxos podem ser obtidos a partir da desodorização de óleos vegetais e da acidulação da borra de neutralização (GONZÁLEZ et al., 1999; FRÉ, 2009).

Os ácidos graxos podem ser usados na alimentação humana, na alimentação animal, na produção de sabões e biodieseis e no tratamento de minérios (BRANDÃO et al., 2005; LEÃO, 2009; FRÉ, 2009).

LEÃO (2009) relata que as propriedades físicas dos ácidos graxos são 
determinadas, principalmente, pelo comprimento e pelo grau de insaturação da cadeia hidrocarbônica. A presença de duplas ligações na cadeia hidrocarbônica faz com que haja uma modificação espacial na cadeia, promovendo seu dobramento no plano. E assim, conferindo um arranjo mais fraco entre as moléculas. Dessa forma, permite uma dissociação mais fácil e um estado físico mais liquefeito em relação a um ácido graxo saturado de número de carbonos correspondente.

O objetivo deste trabalho foi estimar a energia de ativação de fluxo dos ácidos oleico e palmítico a partir da análise de regressão linear de dados da viscosidade absoluta em função da temperatura. Para tanto, estes dados da viscosidade absoluta foram obtidos da equação de Andrade e do modelo de GONZÁLEZ et al. (1999).

\section{MATERIAIS E MÉTODOS}

A Equação 3 corresponde à equação de Andrade (GONZÁLEZ et al., 1999).

$$
\ln \mu=\mathrm{A}+\frac{\mathrm{B}}{\mathrm{T}}
$$

A Tabela 1 relaciona os valores das constantes A e B na equação de Andrade.

Tabela 1 - Valores das constantes da equação de Andrade

\begin{tabular}{ccc}
\hline Ácidos graxos & A & B \\
\cline { 2 - 3 } Oleico & $-5,907$ & $2,760 \times 10^{3}$ \\
Palmítico & $-6,103$ & $2,785 \times 10^{3}$ \\
\hline GONZÁLEZ et al. (1999).
\end{tabular}

A Tabela 2 relaciona os valores obtidos da equação de Andrade para a viscosidade absoluta (em cP) em função da temperatura.

Tabela 2 - Valores da viscosidade absoluta obtidos da equação de Andrade

\begin{tabular}{ccc}
\hline $\begin{array}{c}\text { Temperatura } \\
(\mathrm{K})\end{array}$ & $\begin{array}{c}\text { Viscosidade } \\
(\mathrm{cP})\end{array}$ & $\begin{array}{c}\text { Viscosidade } \\
(\mathrm{cP})\end{array}$ \\
\cline { 2 - 3 } & Ácido oleico & Ácido palmítico \\
\hline 343,15 & 8,467 & 7,486 \\
345,15 & 8,081 & 7,142 \\
347,15 & 7,717 & 6,817 \\
349,15 & 7,374 & 6,511 \\
351,15 & 7,049 & 6,222 \\
353,15 & 6,742 & 5,949 \\
355,15 & 6,452 & 5,690 \\
357,15 & 6,177 & 5,446 \\
359,15 & 5,917 & 5,214 \\
361,15 & 5,670 & 4,995 \\
363,15 & 5,434 & 4,788 \\
365,15 & 5,215 & 4,591 \\
367,15 & 5,005 & 4,404 \\
369,15 & 4,805 & 4,226 \\
371,15 & 4,615 & 4,058 \\
373,15 & 4,435 & 3,898 \\
\hline
\end{tabular}

Fonte: Autor.

GONZÁLEZ et al. (1999) propõem para o ácido oleico a Equação 4 e para o ácido palmítico a Equação 5.

$$
\mu=-7,887 \times 10^{-5} \cdot T^{3}+0,0202 \cdot T^{2}-1,8258 \cdot T+63,16
$$

$$
\mu=17922 \cdot \mathrm{T}^{(-1,83)}
$$

A Tabela 3 relaciona os valores obtidos do modelo de GONZÁLEZ et al. (1999) para a viscosidade absoluta (em cP) em função da temperatura.

Tabela 3 - Valores da viscosidade absoluta obtidos do modelo de GONZÁLEZ et al. (1999)

\begin{tabular}{ccc}
\hline $\begin{array}{c}\text { Temperatura } \\
\left({ }^{\circ} \mathrm{C}\right)\end{array}$ & $\begin{array}{c}\text { Viscosidade } \\
(\mathrm{cP})\end{array}$ & $\begin{array}{c}\text { Viscosidade } \\
(\mathrm{cP})\end{array}$ \\
\cline { 2 - 3 } & Ácido oleico & Ácido palmítico \\
\hline 70 & 7,282 & 7,531 \\
72 & 6,981 & 7,153
\end{tabular}




\begin{tabular}{ccc}
74 & 6,706 & 6,803 \\
76 & 6,452 & 6,479 \\
78 & 6,217 & 6,178 \\
80 & 5,995 & 5,898 \\
82 & 5,783 & 5,638 \\
84 & 5,577 & 5,395 \\
86 & 5,375 & 5,167 \\
88 & 5,171 & 4,954 \\
90 & 4,962 & 4,755 \\
92 & 4,744 & 4,567 \\
94 & 4,514 & 4,391 \\
96 & 4,267 & 4,225 \\
98 & 4,001 & 4,069 \\
100 & 3,710 & 3,921 \\
\hline
\end{tabular}

Fonte: Autor.

A Tabela 4 relaciona os valores da viscosidade absoluta para o ácido oleico em função da temperatura (em unidades do Sistema Internacional de Unidades). Enquanto que a Tabela 5 relaciona os valores da viscosidade absoluta para o ácido palmítico em função da temperatura.

Para a obtenção da razão $\left(E_{a} / R\right)$ e do parâmetro $\ln \mu_{\infty}$ foi realizada uma análise de regressão linear dos dados de $\ln \mu$ versus $(1 / \mathrm{T})$.

A Tabela 6 relaciona os valores de $\ln \mu$ em função de (1/T) para o ácido oleico. Já a Tabela 7 relaciona os valores de $\ln \mu$ em função de (1/T) para o ácido palmítico.

Tabela 4 - Valores da viscosidade absoluta do ácido oleico em função da temperatura

\begin{tabular}{ccc}
\hline $\begin{array}{c}\text { Temperatura } \\
(\mathrm{K})\end{array}$ & $\begin{array}{c}\text { Viscosidade } \\
(\mathrm{Pa} . \mathrm{s})\left(\mathrm{x} 10^{-3}\right)\end{array}$ & $\begin{array}{c}\text { Viscosidade } \\
(\mathrm{Pa} . \mathrm{s})\left(\mathrm{x} 10^{-3}\right)\end{array}$ \\
\cline { 2 - 3 } & Andrade & $\begin{array}{c}\text { González e } \\
\text { coautores }\end{array}$ \\
\hline 343,15 & 8,467 & 7,282 \\
345,15 & 8,081 & 6,981 \\
347,15 & 7,717 & 6,706 \\
349,15 & 7,374 & 6,452 \\
351,15 & 7,049 & 6,217 \\
353,15 & 6,742 & 5,995 \\
355,15 & 6,452 & 5,783
\end{tabular}

\begin{tabular}{lll}
357,15 & 6,177 & 5,577 \\
359,15 & 5,917 & 5,375 \\
361,15 & 5,670 & 5,171 \\
363,15 & 5,434 & 4,962 \\
365,15 & 5,215 & 4,744 \\
367,15 & 5,005 & 4,514 \\
369,15 & 4,805 & 4,267 \\
371,15 & 4,615 & 4,001 \\
373,15 & 4,435 & 3,710 \\
\hline
\end{tabular}

Fonte: Autor.

Tabela 5 - Valores da viscosidade absoluta do ácido palmítico em função da temperatura

\begin{tabular}{ccc}
\hline $\begin{array}{c}\text { Temperatura } \\
(\mathrm{K})\end{array}$ & $\begin{array}{c}\text { Viscosidade } \\
(\text { Pa.s })\left(\times 10^{-3}\right)\end{array}$ & $\begin{array}{c}\text { Viscosidade } \\
(\text { Pa.s }) \times 10^{-3}\end{array}$ \\
\cline { 2 - 3 } & Andrade & $\begin{array}{c}\text { González e } \\
\text { coautores }\end{array}$ \\
\hline 343,15 & 7,486 & 7,531 \\
345,15 & 7,142 & 7,153 \\
347,15 & 6,817 & 6,803 \\
349,15 & 6,511 & 6,479 \\
351,15 & 6,222 & 6,178 \\
353,15 & 5,949 & 5,898 \\
355,15 & 5,690 & 5,638 \\
357,15 & 5,446 & 5,395 \\
359,15 & 5,214 & 5,167 \\
361,15 & 4,995 & 4,954 \\
363,15 & 4,788 & 4,755 \\
365,15 & 4,591 & 4,567 \\
367,15 & 4,404 & 4,391 \\
369,15 & 4,226 & 4,225 \\
371,15 & 4,058 & 4,069 \\
373,15 & 3,898 & 3,921 \\
\hline
\end{tabular}

Fonte: Autor.

Com base nos dados indicados nas Tabelas 6 e 7, foi realizada a análise de regressão linear dos dados de $\ln \mu$ em função de $(1 / \mathrm{T})$.

Tabela 6 - Valores de $\ln \mu$ em função de (1/T) para o ácido oleico

\begin{tabular}{ccc}
\hline$(1 / \mathrm{T})\left(\mathrm{K}^{-1}\right)\left(\mathrm{x} 10^{-3}\right)$ & $\ln \mu$ & $\ln \mu$ \\
\cline { 2 - 3 } & Andrade & González e coautores \\
\hline 2,914 & $-4,772$ & $-4,922$ \\
2,897 & $-4,818$ & $-4,965$ \\
2,881 & $-4,864$ & $-5,005$ \\
2,864 & $-4,910$ & $-5,043$ \\
2,848 & $-4,955$ & $-5,081$ \\
2,832 & $-4,999$ & $-5,117$ \\
2,816 & $-5,043$ & $-5,153$ \\
2,800 & $-5,087$ & $-5,189$ \\
2,784 & $-5,130$ & $-5,226$
\end{tabular}




\begin{tabular}{lll}
2,769 & $-5,173$ & $-5,265$ \\
2,754 & $-5,215$ & $-5,306$ \\
2,739 & $-5,256$ & $-5,351$ \\
2,724 & $-5,297$ & $-5,401$ \\
2,709 & $-5,338$ & $-5,457$ \\
2,694 & $-5,378$ & $-5,521$ \\
2,680 & $-5,418$ & $-5,597$ \\
\hline
\end{tabular}

Fonte: Autor.

Tabela 7 - Valores de $\ln \mu$ em função de (1/T) para o ácido palmítico

\begin{tabular}{ccc}
\hline$(1 / \mathrm{T})\left(\mathrm{K}^{-1}\right)\left(\times 10^{-3}\right)$ & $\ln \mu$ & $\ln \mu$ \\
\cline { 2 - 3 } & Andrade & González e coautores \\
\hline 2,914 & $-4,895$ & $-4,889$ \\
2,897 & $-4,942$ & $-4,940$ \\
2,881 & $-4,988$ & $-4,990$ \\
2,864 & $-5,034$ & $-5,039$ \\
2,848 & $-5,080$ & $-5,087$ \\
2,832 & $-5,125$ & $-5,133$ \\
2,816 & $-5,169$ & $-5,178$ \\
2,800 & $-5,213$ & $-5,222$ \\
2,784 & $-5,256$ & $-5,266$ \\
2,769 & $-5,299$ & $-5,308$ \\
2,754 & $-5,342$ & $-5,349$ \\
2,739 & $-5,384$ & $-5,389$ \\
2,724 & $-5,425$ & $-5,428$ \\
2,709 & $-5,466$ & $-5,467$ \\
2,694 & $-5,507$ & $-5,504$ \\
2,680 & $-5,547$ & $-5,541$ \\
\hline
\end{tabular}

Fonte: Autor.

A análise de regressão linear foi proposta por CANCIAM (2012).

A energia de ativação de fluxo foi determinada pela multiplicação da razão $\left(E_{a} / R\right)$ por $R$. Para este cálculo, foi considerado que a constante dos gases ideais (R) equivale a $8,314 \times 10^{-3} \mathrm{~kJ} \cdot \mathrm{mol}^{-1} \cdot \mathrm{K}^{-1}$.

\section{RESULTADOS E DISCUSSÃO}

A Figura 1 ilustra o gráfico de $\ln \mu$ versus (1/T) para o ácido oleico nos dois modelos matemáticos estudados.
Figura 1: Gráfico $\ln \mu$ versus (1/T) para o ácido oleico nos modelos estudados

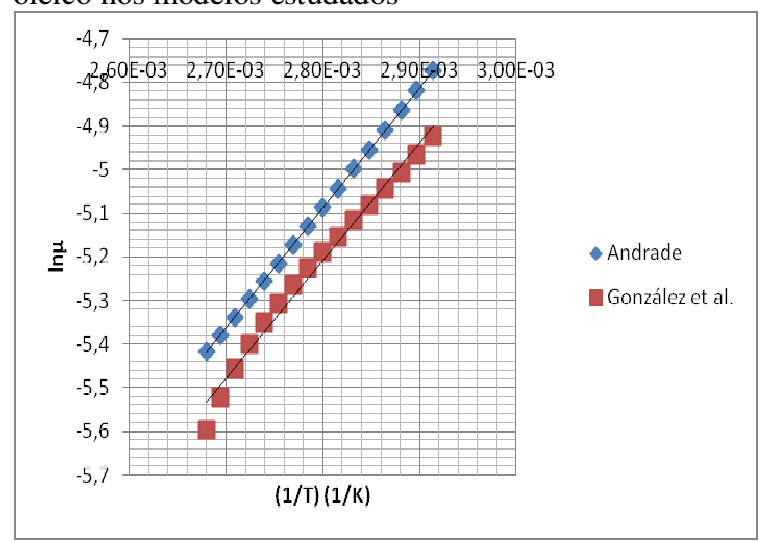

Fonte: Autor.

Já a Figura 2 ilustra o gráfico de $\ln \mu$ versus (1/T) para o ácido palmítico nos dois modelos matemáticos estudados.

Figura 2: Gráfico $\ln \mu$ versus (1/T) para o ácido palmítico nos modelos estudados

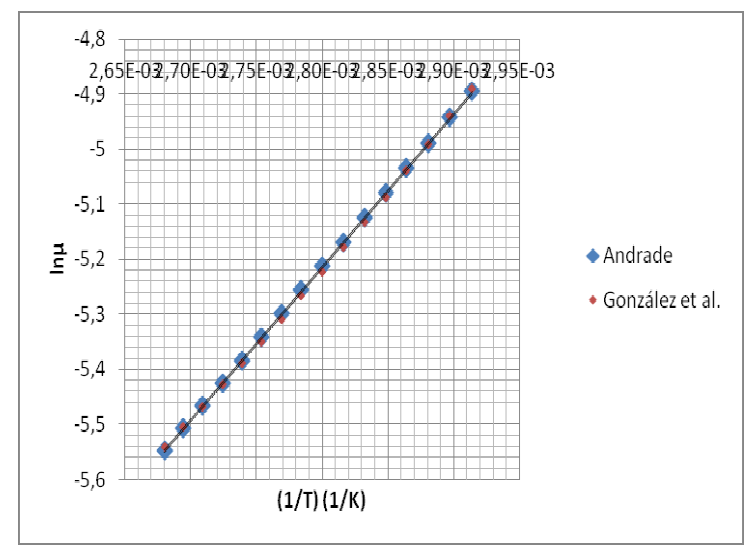

Fonte: Autor.

A Tabela 8 relaciona os resultados obtidos através da análise de regressão linear dos dados de $\ln \mu$ versus (1/T) para o ácido oleico.

A Tabela 9 indica os valores encontrados dos parâmetros $\mu_{\infty}$ e $\mathrm{E}_{\mathrm{a}}$ para o ácido oleico. 
Tabela 8 - Valores da análise de regressão para o ácido oleico

\begin{tabular}{ccc}
\hline Parâmetros & Andrade & González e coautores \\
\hline $\ln \mu_{\infty}$ & $-12,815$ & $-12,779$ \\
$\mathrm{E}_{\mathrm{a}} / \mathrm{R}$ & 2760,126 & 2703,499 \\
$\mathrm{r}^{2}$ & 0,999 & 0,992 \\
\hline
\end{tabular}

Fonte: Autor.

Tabela 9 - Valores dos parâmetros $\mu_{\infty}$ e $E_{a}$ para o ácido oleico

\begin{tabular}{ccc}
\hline Parâmetros & Andrade & González e coautores \\
\hline$\mu_{\infty}($ Pa.s $)$ & $2,720 \times 10^{-6}$ & $2,819 \times 10^{-6}$ \\
$\mathrm{E}_{\mathrm{a}}\left(\mathrm{kJ} \cdot \mathrm{mol}^{-1}\right)$ & 22,948 & 22,477 \\
\hline
\end{tabular}

Fonte: Autor.

Pode-se observar na Tabela 9 que, para o parâmetro $\mu_{\infty}, \quad \mathrm{o}$ modelo de GONZÁLEZ et al. (1999) apresentou maior valor. $\mathrm{O}$ valor do parâmetro $\mu_{\infty}$ pelo modelo de GONZÁLEZ et al. (1999) foi 3,64\% maior em comparação com o valor do parâmetro $\mu_{\infty}$ pelo modelo de Andrade.

Entretanto para a energia de ativação de fluxo, a Tabela 9 revela que o modelo de Andrade apresentou maior valor. O valor da energia de ativação de fluxo pelo modelo de Andrade foi $2,10 \%$ maior em comparação com o valor da energia de ativação de fluxo pelo modelo de GONZÁLEZ et al. (1999).

Quanto aos valores para a energia de ativação de fluxo do ácido oleico, a literatura não dispõe de valores experimentais.

ALVARADO (1995), estudando o efeito da temperatura na viscosidade de óleos vegetais entre 20 e $70^{\circ} \mathrm{C}$, obteve para o óleo bruto de linhaça uma energia de ativação de fluxo equivalente a $22,2 \mathrm{~kJ} \cdot \mathrm{mol}^{-1}$.
Dessa forma, o valor da energia de ativação de fluxo do ácido oleico pelo modelo de Andrade é 3,37\% maior em comparação com o valor da energia de ativação de fluxo do óleo bruto de linhaça. Pelo modelo de GONZÁLEZ et al. (1999), este é 1,25\% maior em comparação com o valor da energia de ativação do óleo bruto de linhaça.

A Tabela 10 relaciona os resultados obtidos através da análise de regressão linear dos dados de $\ln \mu$ versus (1/T) para o ácido palmítico. Enquanto que a Tabela 11 indica os valores encontrados dos parâmetros $\mu_{\infty}$ e $E_{a}$ para este mesmo ácido.

Tabela 10 - Valores da análise de regressão para o ácido palmítico

\begin{tabular}{ccc}
\hline Parâmetros & Andrade & González e coautores \\
\hline $\ln \mu_{\infty}$ & $-13,010$ & $-13,002$ \\
$\mathrm{E}_{\mathrm{a}} / \mathrm{R}$ & 2784,709 & 2780,636 \\
$\mathrm{r}^{2}$ & 0,999 & 0,999 \\
\hline
\end{tabular}

Fonte: Autor.

Tabela 11 - Valores dos parâmetros $\mu_{\infty}$ e $E_{a}$ para o ácido palmítico

\begin{tabular}{ccc}
\hline Parâmetros & Andrade & González e coautores \\
\hline$\mu_{\infty}(\mathrm{Pa} . \mathrm{s})$ & $2,238 \times 10^{-6}$ & $2,256 \times 10^{-6}$ \\
$\mathrm{E}_{\mathrm{a}}\left(\mathrm{kJ} \cdot \mathrm{mol}^{-1}\right)$ & 23,152 & 23,118 \\
\hline Fonte: Autor. & &
\end{tabular}

Pode-se observar na Tabela 11 que, para o parâmetro $\mu_{\infty}, \quad \mathrm{o}$ modelo de GONZÁLEZ et al. (1999) apresentou maior valor. $\mathrm{O}$ valor do parâmetro $\mu_{\infty}$ pelo modelo de GONZÁLEZ et al. (1999) foi 0,80\% maior em comparação com o valor do parâmetro $\mu_{\infty}$ pelo modelo de Andrade. 
Entretanto para a energia de ativação de fluxo, a Tabela 11 indica que o modelo de Andrade apresentou maior valor. $\mathrm{O}$ valor da energia de ativação de fluxo pelo modelo de Andrade foi $0,15 \%$ maior em comparação com o valor da energia de ativação de fluxo pelo modelo de GONZÁLEZ et al. (1999).

Quanto aos valores da energia de ativação de fluxo do ácido palmítico, a literatura não dispõe de valores experimentais para o ácido palmítico.

ALVARADO (1995) obteve para o óleo bruto de abacate, uma energia de ativação de fluxo equivalente a $23,6 \mathrm{~kJ} \cdot \mathrm{mol}^{-1}$.

Assim, o valor da energia de ativação de fluxo do óleo bruto de abacate é $1,94 \%$ maior em comparação com o valor da energia de ativação do ácido palmítico pelo modelo de Andrade. E 2,09\% maior em comparação com o valor da energia de ativação do ácido palmítico pelo modelo de GONZÁLEZ et al. (1999).

Com relação ao coeficiente de correlação $\left(r^{2}\right)$, PINHEIRO et al. (2009) relatam que quanto mais próximo o coeficiente de correlação for da unidade, melhor o ajuste da reta em relação aos pontos da dispersão.

LIRA (2004) comenta que a correlação linear é classificada como muito forte, quando o valor do coeficiente de correlação é maior ou igual a 0,90 e menor que 1,0 .
Assim, observa-se nas Tabelas 8 e 10, que as correlações lineares são classificadas como muito fortes.

Associando a variação da temperatura com a energia de ativação de fluxo, TORALLES et al. (2006) descrevem que, em geral, uma alta energia de ativação de fluxo implica que pequenas variações de temperatura são necessárias para modificar rapidamente a viscosidade absoluta. Assim, de acordo com as Tabelas 9 e 11, os resultados sugerem que o ácido palmítico apresenta uma maior sensibilidade da viscosidade absoluta, em relação ao ácido oleico, quanto à mudança de temperatura.

Essa diferença entre as sensibilidades deve se pequena, uma vez que as energias de ativação de fluxo para o ácidos graxos estudados são próximas.

Pelo modelo de Andrade, a energia de ativação de fluxo do ácido palmítico é $0,89 \%$ maior em comparação com a energia de ativação de fluxo do ácido oleico.

Pelo modelo de GONZÁLEZ et al. (1999), a energia de ativação de fluxo do ácido palmítico é $2,85 \%$ maior em comparação com a energia de ativação de fluxo do ácido oleico.

A diferença entre as sensibilidades da viscosidade absoluta com a mudança de temperatura pode estar associada com a presença da insaturação na cadeia hidrocarbônica e com o número de átomos de carbono. 


\section{CONCLUSÃO}

Os resultados sugerem que o ácido palmítico apresenta uma maior sensibilidade da viscosidade absoluta à mudança de temperatura em relação ao ácido oleico.

Esta diferença entre as sensibilidades deve se pequena, uma vez que os valores das energias de ativação de fluxo são próximos.

Pelo modelo de Andrade, a energia de ativação de fluxo do ácido palmítico é $0,89 \%$ maior em comparação com a energia de ativação de fluxo do ácido oleico.

Pelo modelo de GONZÁLEZ et al. (1999), a energia de ativação de fluxo do ácido palmítico é 2,85\% maior em comparação com a energia de ativação de fluxo do ácido oleico.

Com base nos valores do coeficiente de correlação, as correlações lineares são classificadas como muito fortes.

$\mathrm{Na}$ literatura, foi observada a ausência de valores experimentais para a energia de ativação de fluxo dos ácidos oleico e palmítico.

Entretanto, os resultados sugerem uma proximidade da energia de ativação de fluxo do ácido oleico com a energia de ativação de fluxo do óleo bruto de linhaça.

Enquanto que para a energia de ativação de fluxo do ácido palmítico, existe uma proximidade com a energia de ativação de fluxo do óleo bruto de abacate.

\section{REFERÊNCIAS}

ALVARADO, J. D. Propiedades mecánicas de aceites y grasas vegetales. Grasas y Aceites, v. 46, n. 4-5, p. 264-269, 1995.

BRANDÃO, P. A.; COSTA, F. G. P.; BARROS, L. R.; NASCIMENTO, G. A. J. Ácidos graxos e colesterol na alimentação humana. Agropecuária Técnica, v. 26, n. 1, p. 5-14, 2005 .

BRINGEL, R. M.; SOARES, S. A.; SOARES, J. B. Análise de susceptibilidade térmica de ligantes asfálticos através da energia de ativação de fluxo. Transportes, v.17, n. 1, p. 46-52, 2009.

BRUICE, P. Y. Química Orgânica - volume 2. Pearson Education do Brasil, São Paulo, 2006. 641p.

CANCIAM, C. A. Efeito da temperatura na viscosidade de soluções de sacarose. In:

SANTOS JÚNIOR, G.; ALMEIDA, D. M.; MICHALOSKI, A. O. (Eds). Série em Ciência e Tecnologia de Alimentos: agroindústria, energia e meio ambiente. Ponta Grossa: UTFPR, 2008.

CANCIAM, C. A. Estimativa da energia de ativação de fluxo dos óleos lubrificantes SAE30, SAE40 e SAE50. Revista da Universidade Vale do Rio Verde, v. 10, n. 2, p. 2012-210, 2012.

FASINA, O. O.; COLLEY, Z. Viscosity and specific heat of vegetable oils as a function of temperature: $35^{\circ} \mathrm{C}$ to $180^{\circ} \mathrm{C}$. Internation Journal of Food Properties, v. 11, p. 738-746, 2008.

FRÉ, N. C. D. Obtenção de ácidos graxos a partir da acidulação de borra de neutralização de óleo de soja. Dissertação de Mestrado do Programa de Pós-graduação em Engenharia Química da Universidade Federal do Rio Grande do Sul, 2009. 112p.

GIAP, S. G. E. The hidden property of Arrhenius-type relationship: viscosity as a 
function of temperature. Journal of Physical Science, v. 2, n. 1, p. 29-39, 2010.

GONZÁLEZ, F. O. C.; GONZÁLEZ, M. M. P.; GANCEDO, J. C. B.; SUÁREZ, R. A. Estudio de la densidad y de la viscosidad de algunos ácidos grasos puros. Grasas y Aceites, v. 50, n. 5, p. 359-358, 1999.

GRANJEIRO, A. A.; QUEIROZ, A. J. M.; FIGUEIRÊDO, R. M. F.; MATA, M. E. R. M. C. Viscosidades de polpas concentradas de figo-da-Índia. Revista Brasileira de Agrociência, v. 13, n. 2, p. 219-224, 2007.

GRATÃO, A. C. A.; BERTO, M. I.; SILVEIRA JÚNIOR, V. Reologia do açúcar líquido invertido: influência da temperatura na viscosidade. Ciência e Tecnologia de Alimentos, v. 24, n. 4, p. 652-656, 2004.

KERSTNER, E. K.; SCHAEFFER, L.; LORENZI, M. S.; MERGUTTI, D. M.; ROSADO, C. C. Aspectos reológicos do feedstock para utilização no processo MIM: revisão. Revista Eletrônica de Materiais e Processos, v. 6, n. 1, p. 21-27, 2011.

LEÃO, L. S. Estudo empírico e cinético da esterificação de ácidos graxos saturados sobre o ácido nióbico. Dissertação de Mestrado em Tecnologia de Processos Químicos e Bioquímicos da Universidade Federal do Rio de Janeiro, 2009. 84p.

LIRA, S. A. Análise de correlação: abordagem teórica e de construção dos coeficientes com aplicação. Dissertação de Mestrado do Programa de Mestrado em Ciências da Universidade Federal do Paraná, 2004. 196p.

PINHEIRO, J. I. D.; CUNHA, S. B.; CARVAJAL, S. R.; GOMES, G. C.

Estatística básica: a arte de trabalhar com dados. $1^{\text {a }}$ ed. Elsevier, Rio de Janeiro, 2009. $295 \mathrm{p}$.

RIBEIRO, L. D.; CRUZ, S. R. A.; REIS, R. A. Cálculo de viscosidade de misturas não eletrolíticas. In: Congresso Brasileiro de Engenharia Química em Iniciação Científica,
6, 2005, Campinas. Anais... Campinas: Unicamp, 2005.

SILVA, A. A.; PAREDES, M. L. L.; REIS, R. A. Modelagem de viscosidade de misturas não eletrolíticas. In: Congresso Brasileiro de Engenharia Química em Iniciação Científica, 7. 2007, São Carlos. Anais... São Carlos: UFSCAR, 2007.

TORALLES, R. P.; VENDRUSCOLO, J. L.; VENDRUSCOLO, C. T. Reologia de purê homogeneizado de pêssego: efeito da temperatura e concentração. Brazilian Journal of Food Technology, v. 9, n. 1, p. 1-8, 2006. 